\title{
Reduction of Experimental Canine Myocardial Reperfusion Injury by a Monoclonal Antibody (Anti-Mo1, Anti-CD11b) That Inhibits Leukocyte Adhesion
}

Paul J. Simpson, Robert F. Todd III,“ Joseph C. Fantone,‡ Judith K. Mickelson," James D. Griffin," and Benedict R. Lucchesi With the technical assistance of Matthew D. Adams, Paul Hoff, Karl Lee, and Clare E. Rogers

Departments of Pharmacology, Internal Medicine $\left({ }^{\S}\right.$ Cardiology and ${ }^{*}$ Hematology/Oncology), and ${ }^{\ddagger}$ Pathology, The University of Michigan Medical School, Ann Arbor, Michigan 48109-0010; "Division of Tumor Immunology, Dana-Farber Cancer Institute, Department of Medicine, Harvard Medical School, Boston, Massachusetts 02115

\begin{abstract}
A monoclonal antibody (904) that binds to a leukocyte cell adhesion-promoting glycoprotein, (Mo1; CD11b/CD18) was administered (1 mg/kg, iv.) to open chest anesthetized dogs 45 min after the induction of regional myocardial ischemia. Ischemia was produced by occluding the left circumflex coronary artery (LCX) for $90 \mathrm{~min}$ and then reperfusing for $6 \mathrm{~h}$. There was no difference between control and antibody treated groups with respect to arterial blood pressure, heart rate, or LCX blood flow. Administration of antibody produced no observable effect on circulating neutrophil counts, suggesting that antibody-bound neutrophils were not cleared from the circulation. The mean size of myocardial infarct expressed as percentage of the area at risk of infarction that resulted was reduced by $46 \%$ with anti-Mo1 treatment $(25.8 \pm 4.7 \%, n=8)$ compared to control $(47.6 \pm 5.7 \%, n=8 ; P<0.01)$. The area at risk of infarction was similar between groups. Circulating (serum) antibody excess was confirmed in all 8 anti-Mo1 treated dogs by immunofluorescence analysis. Analysis of ST segment elevation on the electrocardiogram as an indicator of the severity of ischemia suggests that the anti-Mo1 reduces infarct size independent of the severity of ischemia. An additional group of $\operatorname{dogs}(n=5)$ was tested with a control monoclonal antibody of the same subtype (murine IgG1) and was found to produce no significant reduction in myocardial infarct size. Accumulation of neutrophils within the myocardium was significantly attenuated with 904 treatment when analyzed by histological methods. These data demonstrate that administration of anti-Mo1 monoclonal antibody after the induction of regional myocardial ischemia results in reduced myocardial reperfusion injury as measured by ultimate infarct size.
\end{abstract}

\section{Introduction}

Mo1 (CD11b/CD18) is a heterodimeric glycoprotein expressed on the plasma membrane of human neutrophils,

Address reprint requests to Dr. B. R. Lucchesi, Department of Pharmacology, M6322 Medical Sciences I, The University of Michigan Medical School, Ann Arbor, MI 48109-0100.

Received for publication 4 June 1987 and in revised form 19 October 1987.

J. Clin. Invest.

(C) The American Society for Clinical Investigation, Inc.

0021-9738/88/02/0624/06 \$2.00

Volume 81, February 1988, 624-629 mononuclear phagocytes, and null cells (1). It consists of two noncovalently linked polypeptides of $155,000 \mathrm{D}$ (the alpha subunit) and $94,000 \mathrm{D}$ (the beta subunit) (2). Monoclonal antibodies specific for either the alpha or beta subunits of Mol have been demonstrated to inhibit certain neutrophil/monocyte functions: ( $a$ ) the binding of C3bi-opsonized particles (3), and $(b)$ adhesive interactions of neutrophils and monocytes including neutrophil aggregation, monocyte/neutrophil adhesion and spreading on substrates (including vascular endothelium), and chemotaxis (4-8). The leukocytes of individuals who are genetically deficient in their expression of the Mol glycoprotein exhibit an impairment of C3bi-particle binding and leukocyte adhesive interactions $(9,10)$. These observations indicate that the Mol glycoprotein has at least two functional domains: $(a)$ a domain that functions as the plasma membrane receptor for C3bi (CR3), and (b) a domain that promotes the adhesion of myeloid leukocytes (7). Other studies have indicated that exposure of neutrophils and monocytes to inflammatory stimuli in vitro and in vivo results in marked increase in surface Mol expression that is coincident with an increase in leukocyte adhesiveness $(11,12)$. The critical role of Mol in the inflammatory response of activated neutrophils is suggested by the inhibitory effect of anti-Mol monoclonal antibody on neutrophil-mediated endothelial cell injury in a rat lung model of adult respiratory distress syndrome (ARDS) ${ }^{1}$ (13) and neutrophil-dependent vascular injury in skin (14).

A substantial and convincing body of evidence indicates that neutrophils cause damage to the heart when the heart has been subjected to a period of regional ischemia and reperfusion. Studies have demonstrated that if dogs are depleted of circulating granulocytes with a neutrophil specific antiserum (15) or nitrogen mustard (16) before the induction of regional myocardial ischemia and reperfusion, the size of myocardial infarct that results is significantly smaller than in dogs with normal circulating neutrophil counts. There are a number of other studies that have shown that agents that inhibit neutrophil activation and accumulation within the myocardium also result in reduced myocardial infarct size (16-20).

In a previous report, we have identified a specific monoclonal antibody (904) that binds to the alpha subunit (CD1 1b) of both dog and human leukocyte Mol $(7,21,22)$. Antibody 904 is specific for an epitope within the adhesion-promoting

1. Abbreviations used in this paper: ARDS, adult respiratory distress syndrome; HR, heart rate; LCX, left circumflex coronary artery; MAP, mean arterial blood pressure; RPP, rate pressure product; TPT, triphenyltetrazolium chloride. 
domain of Mol (7) and inhibits neutrophil (dog and human) aggregation in vitro stimulated by leukoaggregating factors (phorbol myristate acetate and formyl-methionyl-leucyl-phenylalanine, respectively). The purpose of this investigation was to determine whether in vivo administration of this monoclonal antibody to dogs $\mathbf{4 5} \mathrm{min}$ after the induction of regional myocardial ischemia would effectively reduce myocardial injury upon subsequent reperfusion of the myocardium.

\section{Methods}

Monoclonal antibody. The generation of the murine IgG1 anti-Mol monoclonal antibody (clone 904) was described elsewhere $(7,21)$. The antibody binds to an epitope on the CD1 1b, 155-kD alpha polypeptide of the Mol glycoprotein that is expressed in both human and dog neutrophils $(7,21,22)$. Pharmaceutical-grade anti-Mol monoclonal antibody was supplied by Coulter Immunology (Hialeah, FL) and was purified from aseptically collected murine ascites by ammonium sulfate precipitation and protein A affinity chromatography. Sterile-filtered antibody was diluted to a concentration of $5 \mathrm{mg} / \mathrm{ml}$ in PBS containing either 0.5 or $5 \%$ (wt/vol) normal human serum albumin (HSA diluent). The endotoxin level of the two 904 antibody lots used were $1.92 \mathrm{EU} / \mathrm{mg}$ and $1.54 \mathrm{EU} / \mathrm{mg}$; the corresponding endotoxin levels of the two HSA diluent lots employed were $0.96 \mathrm{EU} / \mathrm{mg}$ and 0.12 EU/mg. A control murine monoclonal IgG1 antibody (Ms IgG1; Coulter Immunology) was prepared in a similar fashion (endotoxin level of $0.38 \mathrm{EU} / \mathrm{mg}$ ).

Preparation of dogs. Adult male mongrel dogs (12-17 kg) were anesthetized with a solution of dial urethane $(0.65 \mathrm{ml} / \mathrm{kg})$ that contains allobarbital, $10 \mathrm{~g} /$ liter; urethane, $400 \mathrm{~g} /$ liter; and monoethylurea, 400 $\mathrm{g} /$ liter. Dogs were respired by positive pressure ventilation with room air. The hearts were exposed through a left thoracotomy in the fifth intercostal space and suspended in a pericardial sling. The left circumflex coronary artery was isolated from surrounding connective tissue proximal to any ventricular branches and instrumented with a calibrated electromagnetic flow probe for the continuous recording of blood flow. Catheters were placed into the aorta via the left carotid artery for blood pressure recording and into the left jugular vein for antibody infusion and blood sampling. The standard limb lead II electrocardiogram was recorded continuously.

Regional myocardial ischemia was produced by occluding the circumflex artery for $90 \mathrm{~min}$ and then reperfusing the myocardium in the presence of a critical stenosis (23). The critical stenosis prevents the development of hemorrhagic infarction and reduces the incidence of reperfusion induced ventricular fibrillation. After reperfusing the myocardium for $6 \mathrm{~h}$, the heart was electrically fibrillated and excised. Using the ex vivo dual perfusion histochemical staining technique previously described (15), the infarct size was assessed as a percentage of the area of the myocardium at risk of infarction as well as a percentage of the total left ventricle. This technique involves perfusion of the cannulated circumflex with $1.5 \%$ triphenyltetrazolium chloride (TPT) solution buffered with $20 \mathrm{mM}$ potassium phosphate (pH 7.4), while simultaneously perfusing the remainder of the coronary circulation with Evan's blue dye introduced into the aorta. Both solutions were delivered to the respective vascular distributions under a constant pressure of $100 \mathrm{mmHg}$ at a temperature of $37^{\circ} \mathrm{C}$ for $5 \mathrm{~min}$. The hearts were then cut into five or six $1-\mathrm{cm}$ thick transverse sections and infarct size was determined planimetrically. The method of quantifying infarct size with TPT has been validated by a number of investigators and has been shown to accurately demarcate viable from nonviable myocardial tissue as determined by the histochemical reaction between TPT and myocardial dehydrogenase enzymes (24-27).

Treatment groups. Initially, dogs were randomly assigned to one of two treatment groups for the assessment of anti-Mol antibody on resulting myocardial infarct size. The two groups were: group I, con- trols, received the vehicle for the monoclonal antibody $(0.5 \%$ or $5 \%$ human serum albumin); and group II, anti-Mol, received antibody (904; Coulter Immunology) $1 \mathrm{mg} / \mathrm{kg}$ infused intravenously over 10 min beginning $45 \mathrm{~min}$ after the induction of regional myocardial ischemia. After the results from the initial two groups were analyzed, a third group of dogs was tested in a nonrandomized fashion with five additional dogs. This third group (antibody control) consisted of five dogs that were treated with a control murine monoclonal antibody (same dose and time) of the same subclass (IgG1; Coulter Immunology) that is unreactive with the Mol glycoprotein on canine neutrophils.

Detection of anti-Mol antibody binding to dog leukocytes. At 0, 85, and $120 \mathrm{~min}$ after induction of ischemia, 4-ml aliquots of venous blood were withdrawn from dogs, placed into EDTA-containing tubes, and centrifuged for $5 \mathrm{~min}$ at $800 \mathrm{~g}$. Plasma was separated from the pellet and was saved for subsequent analysis of residual monoclonal antibody; the cellular pellet was depleted of erythrocytes by ammonium chloride lysis, and the residual leukocytes were analyzed for the presence of preexisting bound anti-Mol antibody by immunofluorescence staining (11). Briefly, 1-2 $210^{6}$ leukocytes were incubated for 30 min at $4^{\circ} \mathrm{C}$ in buffer alone or in buffer containing a saturating concentration of murine anti-Mol antibody. The cells were then washed and incubated for an additional $30 \mathrm{~min}$ at $4^{\circ} \mathrm{C}$ in buffer containing a saturating concentration of fluorescein-conjugated goat anti-mouse immunoglobulin (Tago, Inc., Burlingame, CA). Antibody binding (either as a result of in vivo administration of anti-Mol or after in vitro exposure to additional anti-Mol antibody) to dog neutrophils and monocytes was assessed by flow cytometry after selective gating on these myeloid cells (as determined by $\log$ forward angle versus log right angle light scatter) using a Coulter Epics $C$ flow cytometer. The fluorescence intensity of 5,000 cells/determination was used as a quantitative measure of antibody binding.

Detection of residual anti-Mol antibody in the plasma or sera of treated dogs. To document the administration of anti-Mol monoclonal antibody sufficient to produce antibody excess, samples of plasma (EDTA-anticoagulated blood) or serum were analyzed for the presence of residual anti-Mol antibody. This was assessed by indirect immunofluorescence analysis in which $1 \times 10^{6}$ Mol-positive test cells (calcium ionophore A23187-stimulated human neutrophils [11]) were incubated in buffer containing dog plasma or serum $(1 / 2,1 / 4,1 / 8$, $1 / 16$ dilutions) for $30 \mathrm{~min}$ at $4^{\circ} \mathrm{C}$, and then, after washing, in buffer containing a saturating concentration of fluorescein-conjugated goat anti-mouse immunoglobulin for an additional $30 \mathrm{~min}$ at $4^{\circ} \mathrm{C}$. Antibody binding to test cells was quantitated by flow cytometry as detailed above.

Histological assessment of infarction and neutrophil accumulation within the myocardium. A representative histological section stained with hematoxylin and eosin from each heart was evaluated in a blinded manner (as to the treatment group) for neutrophil accumulation within the border region between infarcted and viable tissue. This was done on a semiquantitative grading scale where values of 0 to 4 were assigned, with 0 representing no inflammatory cell infiltrate and 4 representing the greatest degree of cell infiltration.

Statistical analyses. All data are expressed as mean \pm standard error of the mean. Inasmuch as group I (vehicle controls) and group II (anti-Mol) were initially studied at the same time, statistical analysis between these two groups were made with Student's $t$ test. Since group III (control murine antibody) was not studied at the same time as groups I and II, initial statistical analysis did not include group III. The only statistical analysis involving group III, that was included in this report is a comparison of infarct sizes (IN/AR) among the three groups by one-way analysis of variance (ANOVA) followed by an unpaired Student's $t$ test with alpha error correction by the method of Bonferroni. Values of $P<0.05$ were considered significant. Statistical inferences pertaining to the histological assessment of neutrophil accumulation were tested with a two-sample rank sum test (Mann-Whitney $U$ test) (28). 


\section{Results}

29 dogs were used to assess the effects of anti-Mol monoclonal antibody on the myocardial infarct size that results after regional ischemia and reperfusion. Of these 29 dogs, 21 were included in the final analysis of infarct size. Four dogs were eliminated from the study due to failure to develop objective ischemia as measured by electrocardiographic changes and four dogs (three untreated and one treated with anti-Mol antibody) were eliminated due to ventricular fibrillation.

Effects of treatment on resulting myocardial infarct size. Administration of the anti-Mol antibody reduced myocardial infarct size as a percentage of the area at risk by $46 \%$ compared with control (control $n=8$, IN/AR 47.6 $\pm 5.7 \%$; 904 antibody treated $n=8$, IN/AR $25.8 \pm 4.7 \% ; P<0.01$; Fig. $1 A$ ). Similarly, infarct size was significantly smaller with antibody treatment when infarct size was expressed as a percentage of the total left ventricle (control IN/LV 20.4 $2.4 \%$; 904 IN/LV $10.7 \pm 2.1 \%, P<0.005$ ). The percentage of the left ventricle that was rendered ischemic (area at risk/left ventricle) was similar between treatment groups (control AR/LV 43.1 $\pm 2.8 \%$; $904 \mathrm{AR} / \mathrm{LV} 39.5 \pm 2.3 \%$ ). When infarct size is expressed as a function of the extent of ST segment change on the electrocardiogram ( $\partial \mathrm{ST})$, distinct regression lines are derived for vehicle control $(y=2.41 x+36, r=0.61)$ and 904 antibody treatment $(y=2.30 x+12, r=0.73)$ groups with $y$ representing IN/AR and $x$ representing the change in ST segment $(\mathrm{mV} / 10)$; Fig. 1

A

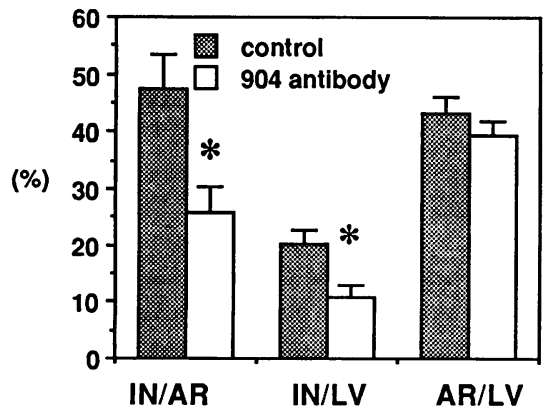

B

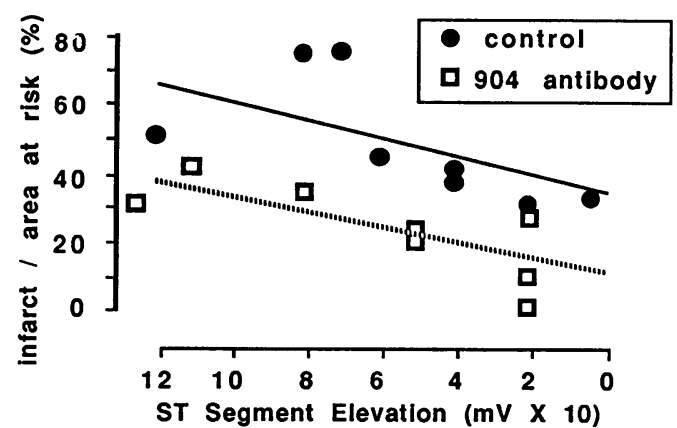

Figure 1. Myocardial infarct size: $(A)$ infarct size depicted as a percentage of the area at risk (IN/AR) or as a percentage of the left ventricle was significantly smaller $\left({ }^{*} P<0.05\right)$ with 904 treatment compared to vehicle control. $(B)$ Infarct/area at risk for individual experiments versus the corresponding ST segment elevation on the electrocardiogram (an indication of the severity of ischemia). Linear regression analysis of the two treatment groups indicate that they scribe different lines with similar slopes. This indicates that infarct size is smaller in the antibody-treated group independent of the severity of the ischemia.
$B$ ). Analysis of changes in ST (JST) segment demonstrated that there was no significant difference between the vehicle control and the 904 antibody-treated groups with respect to the severity of the ischemia (control $\partial \mathrm{ST} 0.48 \pm 0.14 \mathrm{mV} ; 904$ antibody $\partial \mathrm{ST} 0.60 \pm 0.15 ; P=0.598$ ). These data demonstrate that for a given severity of myocardial ischemia (as measured by ST segment elevation), the infarct size that results is larger in the control group compared to the antibody treated group. Thus, myocardial infarct size is reduced by antibody treatment independent of the severity of ischemia. The group that received control murine monoclonal $\operatorname{IgGl}$ antibody $(n=5)$ had infarct sizes that were essentially the same as the vehicle control group (control IgG1; IN/AR $=38.3 \pm 6.1 \%$, IN/LV $=15.2 \pm 2.7 \% ; \mathrm{AR} / \mathrm{LV}=40.1 \pm 2.6 \%$; each not significantly different from vehicle control). When all three experimental groups (control vehicle; 904 antibody; and control monoclonal antibody) were compared simultaneously with ANOVA, we found no statistically significant difference $(P>0.05)$ in myocardial infarct size (IN/AR) between the control vehicle and control monoclonal antibody treated groups nor between the control monoclonal antibody-treated group and the 904 antibody-treated group. However, the control vehicle group was found to be significantly different $(P<0.05)$ from the 904 antibody-treated group when the three groups were analyzed with the ANOVA.

Analysis of residual serum or plasma antibody and antibody binding to leukocytes. By immunofluorescence analysis, the neutrophils of all 29 dogs expressed the antigenic epitope detectable by the anti-Mol monoclonal antibody (not shown). In the nine dogs that received anti-Mol monoclonal antibody, at each time point after antibody administration (40,75, and 405 min after antibody infusion) the serum or plasma contained residual anti-Mol antibody (as assayed by immunofluorescence staining of serum or plasma-treated Mol positive test cells), indicating that an infusion of $1 \mathrm{mg} / \mathrm{kg}$ was sufficient to produce antibody excess in vivo. Detectable subsaturating amounts of bound antibody were found on the leukocytes of only six out of nine dogs which may reflect antibody dissociation from the leukocyte membrane before assay by incubation with the fluorescein conjugated goat anti-mouse immunoglobulin reagent.

Histology. The relative degree of neutrophil accumulation as determined histologically with 0 representing no cellular infiltrate and 4 representing the greatest infiltration score were as follows: Control $(n=8) 3,3,3,3,3,3,2,2 ; 904$ antibody $(n=8)$ $1,1,1,2,2,2,3,4$. Using nonparametric methods to draw statistical inferences (two-sample rank sum test), the 904 antibodytreated group had significantly less neutrophilic infiltrate compared to vehicle controls $(P<0.05)$.

Circulating neutrophil counts. As illustrated, in Fig. 2, by the control group, circulating neutrophils increase in number during the process of myocardial infarction. Treatment with anti-Mol antibody markedly suppressed the early rise in circulating neutrophil count during early reperfusion. However, circulating neutrophil levels did not decrease below baseline levels after antibody treatment.

Hemodynamic effects. Mean arterial blood pressure (MAP), heart rate (HR), left circumflex (LCX) blood flow, and rate pressure product $(\mathrm{RPP}$; MAP $\times \mathrm{HR} / 100)$ were measured (Fig. 3) at regular intervals during the experiments to determine whether the antibody had any effects on these parame- 


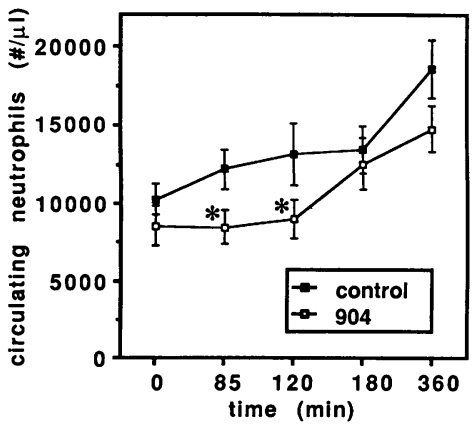

Figure 2. Circulating neutrophil counts. The mean neutrophil counts were significantly less during reperfusion $\left({ }^{*} P<0.05\right)$ in the antibody treated group suggesting that 904 was suppressing the rise in circulating counts. Additionally, the data demonstrate that neutrophils are not cleared from the circulation by treatment with antibody.

ters. The two treatment groups had similar MAP, HR, RPP, and LCX blood flow at baseline and at every time point during the experiments. Thus, the protective effects of the antibody could not be attributed to changes in myocardial blood supply (as measured by LCX blood flow) or myocardial oxygen demand (as measured by RPP or HR or MAP).

\section{Discussion}

In this report we present evidence that the intravenous administration of a monoclonal antibody (904) directed against the leukocyte adhesion promoting molecule, Mol (CD1 lb/CD18) can reduce myocardial reperfusion injury in a well characterized animal model of myocardial infarction. Previous reports have demonstrated that inhibition of neutrophil activation during myocardial infarction with agents that inhibit the fatty acid lipoxygenase enzyme (16-18) or with prostanoids (19, 20). This study extends these observations to indicate that the adhesive interactions of the neutrophil may play a central role in neutrophil mediated damage and that inhibition of neutrophil adhesive interactions with an anti-Mol monoclonal antibody can reduce myocardial reperfusion injury.

Two major determinants of myocardial infarct size are the severity and the duration of the ischemic period. The results from the present study indicate that administration of the
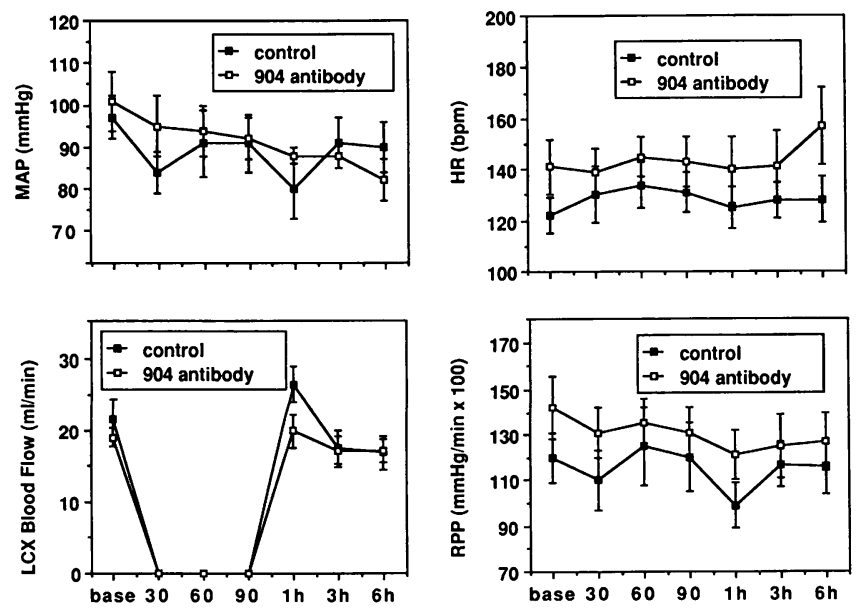

Figure 3. Hemodynamic measurements: MAP, HR, LCX blood flow, and RPP. After baseline (base) measurements were recorded, LCX was occluded for $90 \mathrm{~min}$ and then reperfused for $6 \mathrm{~h}$. There were no significant differences between groups at any time point.

anti-Mol monoclonal antibody (904) provided protection independent of the severity of ischemia as shown in Fig. $1 B$. Inasmuch as the duration of ischemia was $90 \mathrm{~min}$ in all cases, the mechanism of protection by this antibody may be attributed to its inhibitory effects on neutrophil adhesion and spreading within the myocardium.

That the Mol glycoprotein plays a critical part in the inflammatory response of neutrophils is indicated by a number of studies in which monoclonal antibodies directed against Mo1 block adhesion-dependent cellular interactions. AntiMol (anti-CD1 lb or anti-CD18) antibodies inhibit neutrophil aggregation in response to inflammatory stimuli $(6,8,12)$, neutrophil chemotaxis stimulated by chemotactic factors $(7$, 8), and adhesion and spreading of monocytes and neutrophils on substrates including glass, plastic, and monolayers of endothelial and epithelial cells $(4-8,29)$. Additionally, these antibodies inhibit adhesion-dependent neutrophil-mediated damage of endothelial (30) and epithelial monolayers (29) in vitro, and block pulmonary capillary injury induced by activated human neutrophils in a perfused rat lung model of ARDS (13). It has recently been shown that anti-Mol antibody (antiCD18; antibody 60.3) can reduce neutrophil accumulation and plasma leakage in vivo in response to the intradermal injection of the complement activation product, C5a (14) and neutrophil migration to and accumulation in subcutaneously implanted sponges (31). While certain anti-Mol antibodies recognize epitopes within the CR3 binding domain of the Mol glycoprotein and block CR3-dependent cellular interactions (phagocytosis, respiratory burst, and granule release stimulated by particulate activators of the alternative pathway [3, 7 , $8,32,33]$ ), antibody 904 (anti-CD11b) is selective in its inhibitory effect on neutrophil adhesive interactions (specific for an epitope within the adhesion-promoting domain of Mol (7).

Anti-Mol (904) administration in vivo significantly reduced neutrophil accumulation within ischemic myocardial tissue as assessed by histological analysis of tissue sections. These data indicate that neutrophil adhesive interactions are a very important step in neutrophil-mediated myocardial reperfusion injury and that by interfering with neutrophil adhesive interactions, tissue injury may be reduced.

The clinical implications of the present study are profound. For the first time, a neutrophil suppressive agent of great selectivity and specificity with a defined mechanism of action has been shown to be highly effective in inhibiting tissue injury in an experimental model of acute myocardial infarction. Other experimental therapies that suppress neutrophil function and reduce myocardial infarction include neutrophil depletion (15) and the administration of prostacyclin and its analogues $(19,20)$. Prostacyclin and its analogues although very promising in the setting of acute myocardial infarction are not without significant side effects (vasodilation, inhibition of platelet function) and neutrophil depletion is not feasible as a therapeutic intervention. In contrast, the use of a monoclonal antibody (or $F\left(a b^{\prime}\right)_{2}$ fragments) with selective inhibitory effects on neutrophil function offers promise for the future treatment of patients with evolving myocardial infarcts who are candidates for reperfusion by thrombolytic therapy (streptokinase, tissuetype plasminogen activator, pro-urokinase) or by mechanical (balloon angioplasty or coronary artery bypass surgery) means.

There is precedent for the therapeutic use of murine monoclonal antibodies to block leukocyte function in humans. A 
murine IgG1 antibody specific for another adhesion promoting leukocyte glycoprotein, LFA-1 (CD1 1a/CD18; structurally similar to Mol) was administered by iv. infusion $(0.1 \mathrm{mg} / \mathrm{kg}$ per $\mathrm{d}$ every other day for $5 \mathrm{~d}$ ) in allogenic bone marrow transplant patients to block (adhesion dependent) graft versus host disease (34). Additionally, a murine monoclonal IgG1 specific for human leukocyte Fc receptors was used in vivo (monkeys and man) to block clearance of antibody coated erythrocytes (35) or platelets for the treatment of refractory immune thrombocytopenic purpura (36). These data indicate that the use of murine monoclonal antibodies of the $\mathrm{IgG} 1$ subclass directed against Mol may be without serious side effects and may be appropriate for use in humans undergoing acute myocardial infarction as well as other disease processes in which neutrophil-mediated injury is indicated.

\section{Acknowledgments}

The authors thank Drs. Scott Malinconico and Kenneth Kortright (Coulter Immunology, Hialeah, FL) for production of antibodies.

Supported by National Institutes of Health-Heart, Lung, and Blood Institutes grants HL-19782 to Dr. Lucchesi, CA-39064 to Dr. Todd, and HL-32024 and an American Heart Association grant in aid to Dr. Fantone.

\section{References}

1. Todd, R. F., III, L. M. Nadler, and S. F. Schlossman. 1981. Antigens on human monocytes identified by monoclonal antibodies. J. Immunol. 126:1435-1442.

2. Todd, R. F., III, A. van Agthoven, S. F. Schlossman, and C. Terhorst. 1982. Structural analysis of differentiation antigens Mol and Mo2 on human monocytes. Hybridoma. 1:329-337.

3. Arnaout, M. A., R. F. Todd III, N. Dana, J. Melamed, S. F. Schlossman, and H. R. Colten. 1983. Inhibition of phagocytosis of complement $\mathrm{C} 3$ or immunoglobulin G-coated particles and of C3bi binding by monoclonal antibodies to a monocyte-granulocyte membrane glycoprotein (Mo1). J. Clin. Invest. 72:171-179.

4. Harlan, J. M., P. D. Killen, F. M. Senecal, B. R. Schwartz, E. K. Yee, R. F. Taylor, P. G. Beatty, T. H. Price, and H. D. Ochs. 1985. The role of neutrophil membrane glycoprotein GP-150 in neutrophil adherence to endothelium in vitro. Blood. 66:167-178.

5. Wallis, W. J., P. G. Beatty, H. D. Ochs, and J. M. Harlan. 1985. Human monocyte adherence to cultured vasular endothelium: Monoclonal antibody-defined mechanisms. J. Immunol. 135:2323-2330.

6. Wallis, W. J., D. D. Hickstein, B. R. Schwartz, C. H. June, H. D. Ochs, P. G. Beatty, S. J. Klebanoff, and J. H. Harlan. 1986. Monoclonal antibody-defined functional epitopes on the adhesion-promoting glycoprotein complex (CDw18) of human neutrophils. Blood. 67:1007-1013.

7. Dana, N., B. Styrt, J. D. Griffin, R. F. Todd III, M. S. Klempner, and M. A. Arnaout. 1986. Two functional domains in the phagocyte membrane glycoprotein Mol identified with monoclonal antibodies. J. Immunol. 137:3259-3263.

8. Anderson, D. C., L. J. Miller, F. C. Schmalsteig, R. Rothlein, and T. A. Springer. 1986. Contributions of the Mac-1 glycoprotein family to adherence-dependent granulocyte functions. Structure-function assessments employing subunit-specific monoclonal antibodies. J. Immunol. 137:15-27.

9. Dana, N., R. F. Todd III, J. Pitt, T. A. Springer, and M. A. Arnaout. 1984. Deficiency of a surface membrane glycoprotein (Mol) in man. J. Clin. Invest. 73:153-159.

10. Anderson, D. C., and T. A. Springer. 1987. Leukocyte adhesion deficiency: An inherited defect in the MAC-1, LFA-1, and p150,95 glycoproteins. Annu. Rev. Med. 38:175-194.
11. Todd, R. F., III, M. A. Arnaout, R. E. Rosin, C. A. Crowley, W. A. Peters, and B. M. Babior. 1984. Subcellular localization of the large subunit of $\mathrm{Mol}\left(\mathrm{Mol}_{\alpha}\right.$; formerly gp 110), a surface glycoprotein against with neutrophil adhesion. J. Clin. Invest. 74:1280-1290.

12. Arnaout, M. A., R. M. Hakim, R. F. Todd III, N. Dana, and H. R. Colten. 1985. Increased expression of an adhesion-promoting surface glycoprotein in the granulocytopenia of hemodilalysis. $N$. Engl. J. Med. 312:457-462.

13. Ismail, G., M. L. Morganroth, R. F. Todd III, and L. A. Boxer. 1987. Prevention of pulmonary injury in isolated perfused rat lungs by activated human neutrophils preincubated with anti-Mo1 monoclonal antibody. Blood. 69:1167-1174.

14. Arfors, K., C. Lundberg, L. Lindbom, K. Lundberg, P. G. Beatty, and J. M. Harlan. 1987. A Monoclonal antibody to membrane glycoprotein complex CD18 inhibits polymorphonuclear leukocyte accumulation and plasma leakage in vivo. Blood. 69:338-340.

15. Romson, J. L., B. G. Hook, S. L. Kunkel, G. D. Abrams, M. A. Schork, and B. R. Lucchesi. 1983. Reduction of the extent of ischemic myocardial injury by neutrophil depletion in the dog. Circulation. 67:1016-1023.

16. Mullane, K. M., N. Read, J. A. Salmon, and S. Moncada. 1984. Role of leukocytes in acute myocardial infarction in anesthetized dogs: Relationship to myocardial salvage by anti-inflammatory drugs. $J$. Pharmacol. Exp. Ther. 228:510-522.

17. Romson, J. L., B. G. Hook, V. H. Rigot, M. A. Schork, D. P. Swanson, and B. R. Lucchesi. 1982. The effect of ibuprofen on accumulation of 111-indium labelled platelets and leukocytes in experimental myocardial infarction. Circulation. 66:1002-1011.

18. Bednar, M., B. Smith, A. Pinto, and K. M. Mullane. 1985. Nafazatrom-induced salavage of ischemic myocardium in anesthetized dogs is mediated through inhibition of neutrophil function. Circ. Res. 57:131-141.

19. Simpson, P. J., S. E. Mitsos, A. Ventura, K. P. Gallagher, J. C. Fantone, G. C. Abrams, M. A. Schork, and B. R. Lucchesi. 1987. Prostacyclin protects ischemic reperfused myocardium in the dog by inhibition of neutrophil activation. Am. Heart J. 113:129-137.

20. Simpson, P. J., J. K. Mickelson, J. C. Fantone, K. P. Gallagher, and B. R. Lucchesi. 1987. Iloprost inhibits neutrophil function in vitro and in vivo and limits experimental infarct size in the canine heart. Circ. Res. 60:666-673.

21. Letvin, N. L., R. F. Todd III, L. S. Palley, S. F. Schlossman, and J. D. Griffin. 1983. Conservation of myeloid surface antigens on primate granulocytes. Blood. 61:408-410.

22. Giger, U., L. A. Boxer, P. J. Simpson, B. R. Lucchesi, and R. F. Todd III. 1987. Deficiency of leukocyte surface glycoproteins Mol, LFA-1, and Leu M5 in a dog with recurrent bacterial infections. An animal model. Blood. 69:1622-1630.

23. Lucchesi, B. R., W. Burmeister, T. Lomas, and G. Abrams. 1976. Ischemic changes in the canine heart as affected by the dimethylquaternery analog of propranolol UM 272 (SC27761). J. Pharmacol. Exp. Ther. 199:310-328.

24. Roberts, A. J., P. R. Cipriano, D. R. Alonso, J. G. Jacobstein, J. R. Combes, and W. A. Gay. 1978. Evaluation of methods for the quantification of experimental myocardial infarction. Circulation. $57: 35-41$.

25. Fishbein, M. C., S. Meerbaum, J. Rit, U. Lando, K. Kanmatsuse, J. C. Mercier, E. Corday, and W. Ganz. 1981. Early phase of acute myocardial infarct size quantiication: validation of the triphenyltetrazolium chloride technique. Am. Heart J. 101:593-600.

26. Vivaldi, M. T., R. A. Kloner, and F. J. Schoen. 1985. Triphenyltetrazolium staining of irreversible ischemic injury following coronary artery occlusion in rats. Am. J. Pathol. 121:522-530.

27. Romaschin, A. D., I. Rebeyka, G. J. Wilson, and D. A. G. Mickle. 1987. Conjugated dienes in ischemic and reperfused myocardium: an in vivo chemical signature of oxygen free radical mediated injury. J. Mol. Cell. Cardiol. 19:289-302. 
28. Sokal, R. R., and F. J. Rohlf. 1981. Biometry. Second edition. W. H. Freeman Company, New York. pp. 859.

29. Simon, R. H., P. D. DeHart, and R. F. Todd III. 1986. Neutrophil-induced injury of rat pulmonary alveolar epithelial cells. J. Clin. Invest. 78:1375-1386.

30. Diener, A. M., P. G. Beatty, H. D. Ochs, and J. M. Harlan. 1985. The role of neutrophil membrane glycoprotein 150 (GP-150) in neutrophil-mediated endothelial cell injury in vitro. J. Immunol. 135:537-543.

31. Price, T., P. Beatty, and S. Corpuz. 1986. In vivo inhibition of neutrophil function using monoclonal antibody to the CDw18 complex. Clin. Res. 34:467. (Abstr.)

32. Beller, D. I., T. A. Springer, and R. D. Schreiber. 1982. AntiMac-1 selectively inhibits the mouse and human type three complement receptor. J. Exp. Med. 156:1000-1009.

33. Wright, S. D., P. E. Rao, W. C. van Voorhis, L. S. Craigmyle, K.
Iida, M. A. Talle, E. F. Westberg, G. Goldstein, and S. C. Silverstein. 1983. Identification of the C3bi receptor of human monocytes and macrophages by using monoclonal antibodies. Proc. Natl. Acad. Sci. USA. 80:5699-5703.

34. Fisher, A., C. Griscelli, S. Blanche, F. LeDeist, F. Veber, M. Lopez, M. Delaage, D. Olive, C. Mawas, and G. Janossy. 1986. Prevention of graft failure by an anti-HLFA-1 monoclonal antibody in HLA-mismatched bone marrow transplantation. Lancet. ii:10581061.

35. Clarkson, S. B., R. P. Kimberly, J. E. Valinsky, M. D. Witmer, J. B. Bussel, R. L. Nachman, and J. C. Unkeless. 1986. Blockade of clearance of immune complex by an anti-Fc $\gamma$ receptor monoclonal antibody. J. Exp. Med. 164:474-489.

36. Clarkson, S. B., J. B. Bussel, R. P. Kimberly, J. E. Valinsky, J. E. Nachman, and J. C. Unkeless. 1986. Treatment of refractory immune thrombocytopenic purpura with an anti-Fc $\gamma$ receptor antibody. N. Engl. J. Med. 314:1236-1239. 